\title{
Changes in the Structural Features of Organic Matter Extracted from Compost by the Seawater during a 90-day Period under Anaerobic Conditions, and Its Effect on Oogenesis of Brown Macroalga
}

\author{
Hisanori IwaI, ${ }^{* \dagger}$ Yuya TAKASAKI, ${ }^{*}$ and Masami FuKUSHIMA** \\ *Department of Marine System Engineering, Graduate School of Engineering, Osaka Prefecture University, \\ Sakai, Osaka 599-8531, Japan \\ **Division of Sustainable Resource Engineering, Graduate School of Engineering, Hokkaido University, \\ Sapporo 060-8628, Japan
}

\begin{abstract}
Seawater-extractable organic matter (SWEOM) has a potential to serve as an Fe complexing agent in an Fe-fertilizer intended to restore and preserve macroalgal forests. To better understand the restoration technique, structural alterations in SWEOM that occurred during a 90-day period of incubation in seawater were determined. Up to $82 \%$ of the total eluted SWEOM occurred during the initial 30 days; a small amount of SWEOM then continued to elute during the next 60 days. Spectroscopic analyses showed that the high-molecular-weight SWEOM fraction (HMW) altered in aliphaticrich materials, while the low-molecular-weight fraction (LMW) became significantly enriched in aromatic structures. The structural alterations in the HMW and LMW could be caused by the action of anaerobic microorganisms. In addition, approximately 30 and $60 \%$ of the gametophytes were converted to eggs in the presence of HMW and LMW with Fe, respectively.
\end{abstract}

Keywords SWEOM, structural alteration, seawater, seaweed beds, restoration, oogenesis

(Received September 10, 2017; Accepted November 29, 2017; Published April 10, 2018)

\section{Introduction}

Macroalgal forests, corresponding to seaweed beds, play important roles in protecting ecosystems in coastal areas and in supplying useful materials for our society. ${ }^{1,2}$ However, macroalgal deforestation has recently become a serious worldwide problem in coastal areas., ${ }^{3,4}$ The presence of dissolved iron can limit the growth of seaweed. For example, the reproductive growth of brown macroalgae is regulated by iron, ${ }^{5,6}$ and the biomass productivity for brown macroalgal sporophytes is significantly increased when higher levels of dissolved iron are present. ${ }^{7}$ A lack of dissolved iron, then, can cause critical damage to the preservation of seaweed beds. It is known that dissolved organic matter plays important roles in the water solubility and bioavailability of trace levels of iron in seawater. ${ }^{6,8}$ An iron enrichment technique by fertilizing the sea bottom with a mixture consisting of steelmaking slag and compost was recently evaluated for use as a source of dissolved iron in an attempt to recover and preserve seaweed beds in Japan. ${ }^{9}$ Regarding this technique, it was reported that seawaterextractable organic matter (SWEOM) derived from a compost could play an effective role in supplying bioavailable iron for the reproduction of a brown macroalga. ${ }^{6}$ However, in these previous reports, the SWEOM fraction that had been extracted

$\dagger$ To whom correspondence should be addressed.

E-mail: h-iwai@marine.osakafu-u.ac.jp after an incubation period of only 3 days was investigated. ,, $10-12^{-12}$ Regarding developing an understanding of the role for compost in the fertilization of coastal water, the elution characteristics and structural alterations of SWEOM from compost during a longer extraction period under anaerobic seawater conditions should also be investigated.

In this study, the characteristics of SWEOM were monitored during a 90-day incubation period. Because the structural features of SWEOM vary depending on the molecular size, ${ }^{11,12}$ the structural alterations of SWEOM that had been incubated for various periods of time were investigated for the two molecular size fractions above (HMW) and below $500 \mathrm{Da}$ (LMW) by spectrometric analyses. Moreover, the effect of SWEOM on suppling Fe to brown macroalgae was elucidated based on the reproductive growth of female gametophytes, Saccharina japonica (i.e., oogenesis).

\section{Experimental}

Compost sample and artificial seawater

A compost sample, which was prepared from maturing a mixture of bark tips of soft- and hardwood timbers from forest thinning and cowpats obtained from Mori Industry Co., Ltd. (Obihiro, Hokkaido, Japan), were freeze-dried and passed through a 2-mm mesh stainless-steel sieve. Compost powder of less than $2 \mathrm{~mm}$ in size was used in all experiments. The elemental composition of this powder was as follows: C, 38.8; 
H, 4.86; N, 2.45; S, 0.43; O, 35.5; ash, 18.0 (wt \%). The artificial seawater was prepared by dissolving the following salts $(\mathrm{g})$ in $1 \mathrm{~kg}$ of ultrapure water: $\mathrm{NaCl}, 28.5 ; \mathrm{MgSO}_{4} \cdot 7 \mathrm{H}_{2} \mathrm{O}, 7.00$; $\mathrm{MgCl}_{2} \cdot 6 \mathrm{H}_{2} \mathrm{O}, 5.00 ; \mathrm{CaCl}_{2}, 1.11 ; \mathrm{KCl}, 0.73 ; \mathrm{H}_{3} \mathrm{BO}_{3}, 0.03$. Prior to use in the experiment, the $\mathrm{pH}$ of the artificial seawater was adjusted to $8.0-8.1$ using dilute aqueous $\mathrm{HCl}$ and $\mathrm{NaOH}$.

\section{Sequential extraction and purification of SWEOMs}

A 75.0-g portion of dry compost powder and a 750-g portion of artificial seawater $(\mathrm{pH}=8.0, I=0.7)$ were mixed in a $2-\mathrm{L}$ capped Erlenmeyer poly-flask at a solid:liquid ratio of 1:10 (wt/wt). After purging with a $\mathrm{N}_{2}$ gas for $30 \mathrm{~min}$, the flask was sealed tightly and shaken with mechanical shaker (MMS-210, EYELA) for 90 days in the dark. At 3, 6, 9, 12, 15, 27, 39, 60 and 90 days of extraction, the suspension was separated into the liquid and solid phases by centrifugation (10000 rpm, $15 \mathrm{~min}$ ). The solid phase was mixed with newly prepared artificial seawater, and the extraction procedure was then continued after purging the suspension with $\mathrm{N}_{2}$ gas for $30 \mathrm{~min}$. To investigate the elution behavior of the SWEOM, an aliquot of this liquid phase was filtered through 5A filter paper (ADVANTEC), and the total organic carbon (TOC) concentration of this filtrate was measured by means of a TOC analyzer (TOC-V CSH/CSN, Shimadzu). SWEOM fractions were collected from the liquid phase at 3, 15, 27 and 90 days, and were divided into two fractions, HMW and LMW, by means of ultrafiltration (MWCO $500 \mathrm{Da}$ ). SWEOMs were purified according to previous reports. ${ }^{10-12}$ The sequential extraction procedure was duplicated. These purified SWEOMs were freeze-dried and stored in powdered form at $-30^{\circ} \mathrm{C}$ until used. The yields of SWEOMs were evaluated as the value calculated by dividing the obtained powdered fractions $(\mathrm{mg})$ by initially taken compost $(\mathrm{kg})$ and the interval days to seawater exchange (day). To distinguish between samples, the extraction period (number of days) of each SWEOMs are expressed by the number at the end of each fraction label (e.g., HMW3 or LMW90).

\section{Molecular weights}

The molecular weights of the SWEOMs were estimated by HPSEC using a PU-2080-type HPLC system (JASCO) with a TSK-Gel $\alpha$-M column (7.8 mm i.d. $\times 300 \mathrm{~mm}$, Tosoh, Japan) as reported previously. $2600 \mathrm{kDa}, 990 \mathrm{kDa}, 350 \mathrm{kDa}, 150 \mathrm{kDa}$, $77 \mathrm{kDa}, 49 \mathrm{kDa}, 32 \mathrm{kDa}, 17 \mathrm{kDa}, 13 \mathrm{kDa}, 6800 \mathrm{Da}, 4300 \mathrm{Da}$, $1400 \mathrm{Da}$ and $208 \mathrm{Da}$ sized polystyrene sulfonic acid sodium salts were used as standards for calibrating the molecular weight, in which a standard of $2600 \mathrm{kDa}$ was used for determining the void volume $(6.3 \mathrm{~mL})$. Sample solutions were prepared by diluting a $300-\mu \mathrm{L}$ aliquot of a $1000 \mathrm{mg} \mathrm{L}^{-1}$ SWEOM solution to $250 \mathrm{mg} \mathrm{L}^{-1}$ with phosphate buffer $(\mathrm{pH}$ 7.0). The HPSEC conditions were as follows: the mobile phase was a mixture of $0.01 \mathrm{M}$ phosphate buffer $(\mathrm{pH}$ 7.0) and acetonitrile mixture $(75: 25, \mathrm{v} / \mathrm{v})$ at a flow rate of $0.75 \mathrm{~mL} \mathrm{~min}^{-1}$, a column temperature of $40^{\circ} \mathrm{C}$ and a detection wavelength of 260 $\mathrm{nm}$. The weight average $\left(M_{\mathrm{w}}\right)$ and number average $\left(M_{\mathrm{n}}\right)$ molecular weights were calculated using the following equations: ${ }^{13}$

$$
\begin{aligned}
& M_{\mathrm{w}}=\frac{\sum\left(M_{\mathrm{i}} \times h_{\mathrm{i}}\right)}{\sum h_{\mathrm{i}}}, \\
& M_{\mathrm{n}}=\frac{\sum h_{\mathrm{i}}}{\sum\left(h_{\mathrm{i}} / M_{\mathrm{i}}\right)},
\end{aligned}
$$

where $M_{\mathrm{i}}$ and $h_{\mathrm{i}}$ represent the values of the molecular weight and the peak intensity at the corresponding retention times, respectively.

\section{Spectrometric analyses}

UV-vis spectroscopic analysis was performed on a V-630 spectrophotometer (JASCO), using $1 \times 1 \mathrm{~cm}$ quartz cuvettes. The concentration of the samples was $50 \mathrm{mg} \mathrm{L}^{-1}$, which were prepared by diluting $1000 \mathrm{mg} \mathrm{L}^{-1}$ in $0.05 \mathrm{M} \mathrm{NaOH}$ of the SWEOM stock solution with a $0.05 \mathrm{M}$ phosphate buffer solution $(\mathrm{pH} 7.00 \pm 0.05)$. The absorptivities at $600 \mathrm{~nm}\left(\varepsilon_{600}\right)$ and $280 \mathrm{~nm}\left(\varepsilon_{280}\right)$ were calculated using the following equation: ${ }^{14}$

$$
\varepsilon_{600 \text { or } 280}=\frac{\text { Absorbance at } 600 \text { or } 280 \mathrm{~nm}\left(\mathrm{~cm}^{-1}\right)}{[\text { SWEOM }]\left(\mathrm{g} \mathrm{L}^{-1}\right) \times \% \mathrm{C} / 100}
$$

where \%C represents the percent of carbon contents of SWEOMs estimated by TOC analysis.

The FT-IR spectra were recorded on pellets made from a 1:100 (w/w) mixture of dried compost or SWEOM/KBr, using an FT/IR 4100 spectrophotometer (JASCO).

Solid-state CP-MAS ${ }^{13} \mathrm{C}$ NMR spectra were recorded using a Bruker MSL-400-type NMR spectrometer at a field strength corresponding to $75.47 \mathrm{MHz}$ for ${ }^{13} \mathrm{C}$ and $300.5 \mathrm{MHz}$ for ${ }^{1} \mathrm{H}$ using a 4-mm CP MAS probe. The variable amplitude CPMAS technique was applied at a contact time of $1 \mathrm{~ms}$, a spinning speed of $9 \mathrm{kHz}$, a pulse delay of $6 \mathrm{~s}$, and integration times of 8000 .

\section{Gametogenesis assay}

An aseptic stock of female gametophytes of Saccharina japonica was obtained from the Muroran Marine Station, Hokkaido University (Hokkaido, Japan). ${ }^{5}$ A 30-mL aliquot of $\mathrm{ASP}_{12}$, excepting EDTA and $\mathrm{Fe}$, was pipetted into $100-\mathrm{mL}$ Erlenmeyer glass flasks that had been acid-washed and heatsterilized. The 600 or $1200 \mu \mathrm{L}$ of SWEOM stock solution (1000 $\mathrm{mg} \mathrm{L}^{-1}$ in $0.05 \mathrm{M} \mathrm{NaOH}$ ) was added to the medium. Based on the results of a previous study, ${ }^{6}$ the concentrations of HMW and LMW in the media were adjusted at 5 and $10 \mathrm{mg} \mathrm{L}^{-1}$ as being suitable values, respectively. Subsequently, 0 or $30 \mu \mathrm{L}$ of the $1 \mathrm{mM} \mathrm{Fe}^{2+}$ stock solution was added to the resulting media. After sealing with Al-foil, the media were sterilized by autoclaving $\left(121^{\circ} \mathrm{C}, 20 \mathrm{~min}\right)$. After cooling the media to room temperature, the media were inoculated with small fragmented gametophytes under HEPA conditions. The inoculated gametophytes were cultured for 14 days at $10^{\circ} \mathrm{C}$ under a $14 / 10 \mathrm{~h}$ of light/dark cycle in a light condition of $20-40 \mu \mathrm{m}$ photons $\mathrm{m}^{-2} \mathrm{~s}^{-1}$ with cool white LED lights. After a 14-day incubation period, the degree of reproductive growth was estimated to be maturity $(\%)$, which was calculated as the percentage of mature cells. ${ }^{6,7}$

\section{Results and Discussion}

\section{Quantitative change of SWEOM}

To investigate the elution behavior of the SWEOM, TOC values of filtered artificial seawater after extraction for an arbitrary incubation period were measured. In this experiment, artificial seawater was sometimes changed to a new one. The TOC values were standardized by the interval period to a seawater exchange accordingly. Figure 1 shows the kinetical change of the TOC values (mg L $\mathrm{m}^{-1} \mathrm{day}^{-1}$ ) for a 90 day period of incubation. As shown in Fig. 1, the elution of the SWEOM was significantly decreased after 3 days, and reached a plateau after a 27-day incubation period. Most of the SWEOM was eluted during the initial 15 days ( $c a .82 \%$ of the total eluted SWEOM during 90 days); however, a small amount of SWEOM (ca. 2 - 4 mg-C L ${ }^{-1}$ day $^{-1}$ ) continued to elute during the 27 to 90 -day 
period. The yields of SWEOMs are summarized in Table 1. Although the yield of LMW at the initial stage of incubation (3 days) was $496 \mathrm{mg} \mathrm{kg}^{-1}$ day $^{-1}$, the values significantly decreased to 101,27 and $8.3 \mathrm{mg} \mathrm{kg}^{-1}$ day $^{-1}$ for 15,27 and 90 days of incubation periods. The yields of HMWs were also significantly decreased from the initial $\left(142 \mathrm{mg} \mathrm{kg}^{-1} \mathrm{day}^{-1}\right)$ to the end satges ( $c a .6 \mathrm{mg} \mathrm{kg}^{-1} \mathrm{day}^{-1}$ ) of incubation. The yield ratios of HMW:LMW at day 3 and after 15 were about 2:8 and $4: 6$, respectively. It was predicted that LMW was the dominant SWEOM fraction in an initial compost, and that most of the LMW was eluted during the initial stages of the incubation period. However, the amount of the LMW fraction was larger than that of the HMW fraction in any extraction period.

\section{Qualitative changes of SWEOM}

The $M_{\mathrm{w}}$ values and the $M_{\mathrm{w}} / M_{\mathrm{n}}$ ratios are summarized in Table 1 . The values of $M_{\mathrm{w}}$ and $M_{\mathrm{w}} / M_{\mathrm{n}}$ for HMWs significantly increased with increasing incubation period (Table 1) The $M_{\mathrm{w}}$ and $M_{\mathrm{w}} / M_{\mathrm{n}}$ values for the LMWs were not significantly changed because the LMWs were fractions that passed through the ultrafiltration membrane.

\section{Spectroscopic characteristics}

Figure 2 shows UV-vis spectra for the HMWs and LMWs. The spectra for the SWEOMs appeared as broad peaks, which are characteristic of humic substances. For the HMWs, the absorbances decreased gradually during the initial 30-day incubation period; the absorbance for the HMW90 exceeded that for the HMW15. In contrast, for LMWs, the absorbances gradually increased with increasing incubation period. Figure 3 shows the absorptivities at $600 \mathrm{~nm}\left(\varepsilon_{600}\right)$ and $280 \mathrm{~nm}\left(\varepsilon_{280}\right)$, respectively. The values of $\varepsilon_{280}$ and $\varepsilon_{600}$ are related to the degree of conjugated aliphatic polyaromatic structure and that of

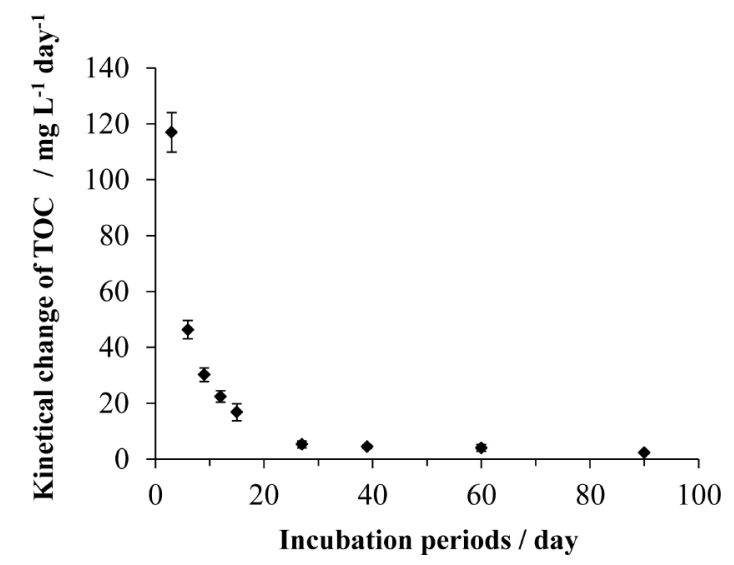

Fig. 1 SWEOM elution behavior during the 90-day extraction period. Plots and error bars represent average values and standard deviations $(n=2)$, respectively. aromaticity, respectively. ${ }^{14}$ In the case of the HMWs, the $\varepsilon_{280}$ and $\varepsilon_{600}$ values decreased with increasing period of extraction, indicating that the content of conjugated bonds and aromatic structures decreased with increasing extraction period. In the case of the LMWs, the two indexes were increased with increasing incubation period, indicating that the content of the aromatic structures was significantly increased.

Figure 4 shows FT-IR spectra for HMWs, LMWs and compost used in the extraction, respectively. The spectral bands were assigned as follows: ${ }^{15-17}$ A broad peak at $3400 \mathrm{~cm}^{-1}$ for intermolecular bonded phenolic $\mathrm{O}-\mathrm{H}$ stretching; a spectral band at $3300 \mathrm{~cm}^{-1}$ for aliphatic N-H stretching; two peaks at $2850-2950 \mathrm{~cm}^{-1}$ for asymmetrical and symmetrical stretching of aliphatic $\mathrm{C}-\mathrm{H}$; a peak or shoulder at $1720 \mathrm{~cm}^{-1}$ for carboxylic $\mathrm{C}=\mathrm{O}$ stretching; a peak at $1650 \mathrm{~cm}^{-1}$ for $\mathrm{C}=\mathrm{O}$ stretching of amide-I band; a peak or shoulder at $1620 \mathrm{~cm}^{-1}$ for aromatic $\mathrm{C}=\mathrm{C}$ stretching; a peak at $1540 \mathrm{~cm}^{-1}$ for $\mathrm{N}-\mathrm{H}$ bending and $\mathrm{C}-\mathrm{N}$ stretching of amide-II band; a peak at $1390 \mathrm{~cm}^{-1}$ for symmetric stretching of carboxylate or aldehydic $\mathrm{C}-\mathrm{H}$ bending; a peak or shoulder band at $1220-1230 \mathrm{~cm}^{-1}$ for phenolic or carboxylic $\mathrm{C}-\mathrm{O}$ stretching and carboxylic or alcoholic $\mathrm{O}-\mathrm{H}$ bending; a spectral band at $1100-1170 \mathrm{~cm}^{-1}$ for $\mathrm{C}-\mathrm{O}-\mathrm{C}$ stretching of ethers or an aliphatic $\mathrm{C}-\mathrm{OH}$ stretching; a peak at $1050 \mathrm{~cm}^{-1}$ for $\mathrm{C}-\mathrm{O}$ stretching of polysaccharides; a broad peak at about $800 \mathrm{~cm}^{-1}$ for out-of-plane N-H wagging of amines.

The peaks at around $3390-3290,2930,1650$ and $1050 \mathrm{~cm}^{-1}$ were conspicuous characteristics of the spectra of the HMWs.

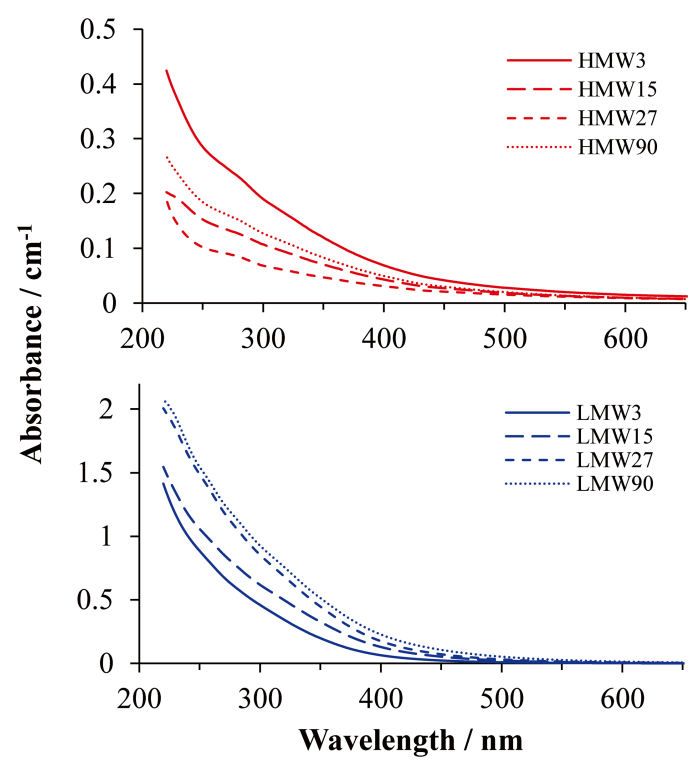

Fig. 2 UV-vis spectra for sequentially extracted SWEOMs. Red and blue lines represent HMWs and LMWs, respectively. Solid, long dashes, short dashes and dotted lines represent 3, 15, 27 and 90 day incubation periods, respectively.

Table 1 Yields and molecular weights of sequentially extracted SWEOMs

\begin{tabular}{|c|c|c|c|c|c|c|c|c|}
\hline & \multicolumn{4}{|c|}{ HMW } & \multicolumn{4}{|c|}{ LMW } \\
\hline & 3 & 15 & 27 & 90 & 3 & 15 & 27 & 90 \\
\hline Yields/mg kg ${ }^{-1}$ day $^{-1}$ & $142 \pm 45$ & $60.5 \pm 21.5$ & $16.0 \pm 2.7$ & $5.75 \pm 0.88$ & $496 \pm 35$ & $101 \pm 37$ & $27.1 \pm 13.7$ & $8.30 \pm 0.92$ \\
\hline Molecular weights $M_{\mathrm{w}}{ }^{\mathrm{a}}$ & 3800 & 5200 & 9000 & 54000 & 1200 & 1500 & 1500 & 2000 \\
\hline$M_{\mathrm{w}} / M_{\mathrm{n}}{ }^{\mathrm{b}}$ & 1.63 & 2.37 & 2.8 & 5.67 & 1.32 & 1.38 & 1.39 & 1.68 \\
\hline
\end{tabular}

a. $M_{\mathrm{w}}$ : Weight-average molecular weight. b. $M_{\mathrm{n}}$ : Number-average molecular weight. $M_{\mathrm{w}} / M_{\mathrm{n}}$ : Polydispersity. 
The broad peak around $3400 \mathrm{~cm}^{-1}$, and the peaks at 1720 and $1220 \mathrm{~cm}^{-1}$ were characteristic in the spectra for the LMWs For the HMWs, the intensity of the peaks at 3290, 1650 and $1541 \mathrm{~cm}^{-1}$ increased with increasing extraction period. This result suggests that the content of nitrogen containing compounds increased during incubation. The intensity of the peak at $2928 \mathrm{~cm}^{-1}$ for the HMWs gradually increased from initial to the later extraction stages, suggesting that the structure of the HMW at the later stage of incubation had a more aliphatic character than that of HMW at the initial stage. In the spectra for LMWs (Fig. 4(B)), the band at $3422 \mathrm{~cm}^{-1}$ and the shoulder at $1620 \mathrm{~cm}^{-1}$ were enhanced during the extraction period, indicating that the content of phenolic and aromatic compounds increased with

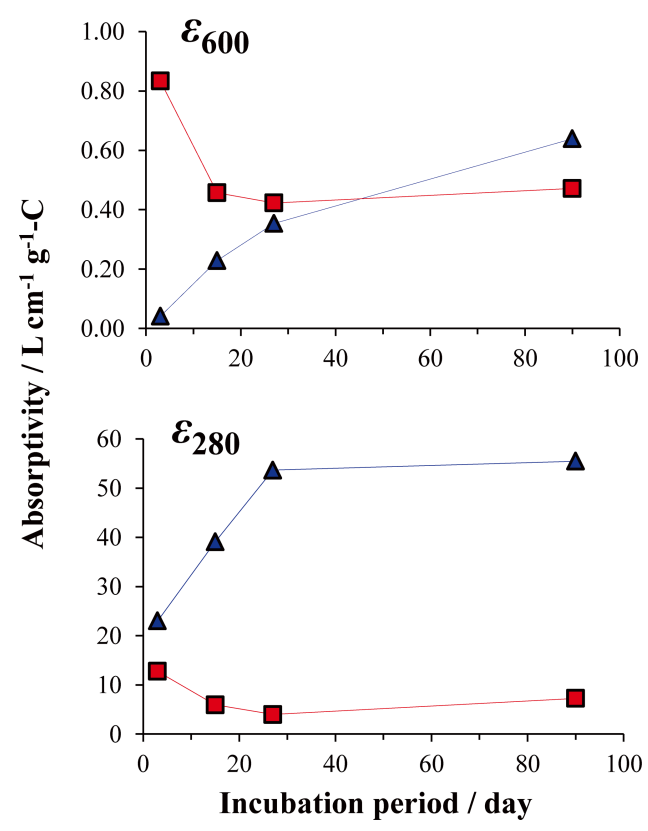

Fig. 3 Changes in the $\varepsilon_{600}$ and $\varepsilon_{280}$ values $\left(\mathrm{L} \mathrm{cm}^{-1} \mathrm{~g}^{-1}-\mathrm{C}\right)$ for sequentially extracted SWEOMs during the 90-day incubation period. Square and triangle plots represent HMW and LMW, respectively. increasing incubation period. The sharp peak at $1719 \mathrm{~cm}^{-1}$ for the LMWs became smaller with increasing extraction period, while the peaks around $1100-1170 \mathrm{~cm}^{-1}$ increased. This trend indicates that carboxylic acid and ether or aliphatic-OH structure were decreased and increased, respectively, during the extraction period. Because compost is a mixture of various types of organic matter, like humic and non-humic substances, the spectra for the composts contained some broad peaks (Fig. 4(C)). Compared to the spectrum for the compost before extraction, the peaks at around 1600, 1220 and $1032 \mathrm{~cm}^{-1}$ were decreased after extraction. Also, a small peak at $796 \mathrm{~cm}^{-1}$ was visible before the extraction was no longer present after the extraction. These results indicated that the composition level of aromatic compounds, carboxylic or hydroxyl groups, saccharides and amines were decreased by the extraction during the 90 days.

Solid-state CP-MAS ${ }^{13} \mathrm{C}$ NMR spectra for the SWEOMs are shown in Fig. 5. The chemical shifts were assigned according to Warshaw et al. ${ }^{18}$ Spectra for the HMWs (Fig. 5 left) show strong peaks assigned to alkyl-C, polysaccharide-C, carbonyl-C, and only a few peaks corresponding to the aromatic- $\mathrm{C}$ region were observed. No significant alteration was evident, except that the peak assigned to polysaccharide-C decreased with increasing incubation period. Spectra for the LMWs (Fig. 5 right) show broad spectral peaks assigned to alkyl-C and small peaks in the region corresponding to carbonyl-C. In the LMW15, 27 and 90 samples, an inconspicuous peak at 120 $140 \mathrm{ppm}$ in the LMW3 change to large peaks, indicating that the aromaticity of the LMWs increased during the incubation. The relative abundance $(\%)$ of carbon species were calculated by integrating the NMR spectral peaks, and are summarized in Table 2. With increasing period of incubation from 3 to 90 days, for the HMWs, the relative abundance of alkyl-C increased by 1.5 , while that of aromatic-C and carbonyl-C decreased by 0.7 . For the LMWs, the relative abundance of aromatic-C increased by 1.6 , while that of polysaccharide-C and carbonyl-C decreased by 0.8 and 0.5 , respectively. In particular, the abundance of aromatic-C attached to hydrogen or carbon $(110-140 \mathrm{ppm})$ for the LMW increased significantly by 1.94 times during the 90 day extraction period.
(A)

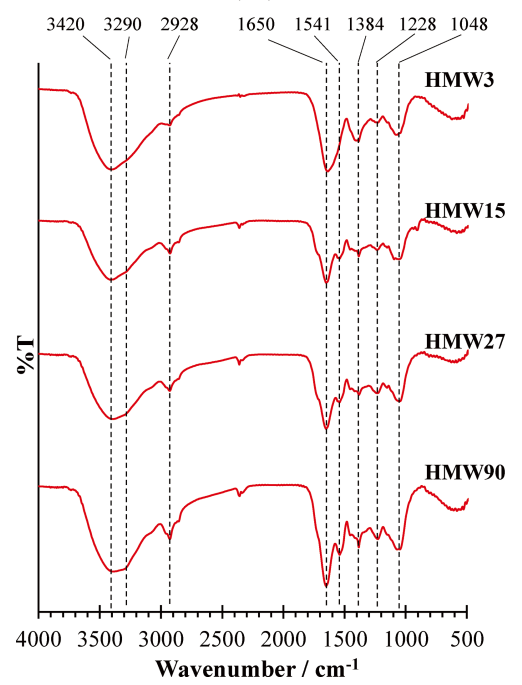

(B)

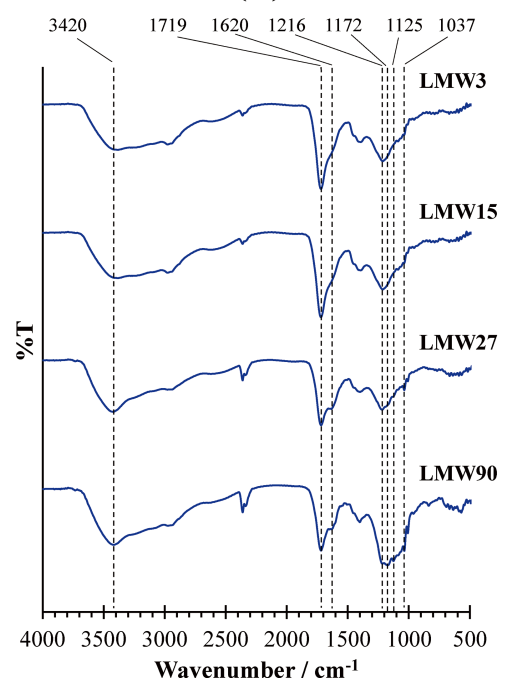

(C)

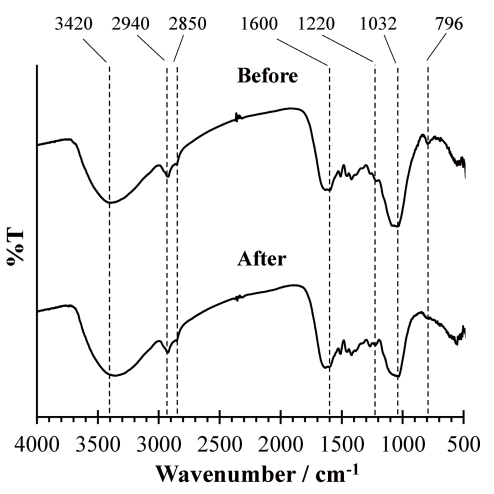

Fig. 4 FT-IR spectra for sequentially extracted SWEOMs and a bark compost. (A), (B) and (C) represent the spectra for HMW, LMW and compost used in this study. 


\section{Summary of structural alterations}

A significant increase in the $M_{\mathrm{w}} / M_{\mathrm{n}}$ values observed for the HMWs indicates that they were comprised of a mixture of macromolecules with a range of molecular size, a significant increase in the $M_{\mathrm{w}}$ of the HMWs, then, resulted from aggregation stimulated by the relatively high concentratioin of divalent cations in seawater. ${ }^{6,19,20}$ The previous study observed that the flocculation of HMW increased during 14 day of incubation in the artificial seawater medium. ${ }^{6}$ The long interval periods to seawater exchange for HMW27 (12 day) and HMW90 (30 day) could induced the aggregation of eluted HMW. It is known that the darkening of humic substances color is attributed to the development of polycondensation and conjugate bond systems. From the results of UV-vis spectral features, structural alterations in HMW and LMW resulted from enrichment in saturated bond and aromatic compounds, respectively. This result is in agreement with those obtained in Figs. 3 - 5. A previous study reported that structural alterations of humic acids that were incubated with coastal seawater might be caused by the activation of anaerobic microorganisms. ${ }^{13}$ It is known that nonaromatic carbohydrates can be converted into substances containing phenolic groups, like lignin monomers, via aromatization through a dehydration process and the shikimic acid pathway. ${ }^{17}$ These aromatization reactions require the enzymatic assistance of anaerobic bacteria, such as Escherichia coli bacteria. ${ }^{17,21}$ The concentration of saccharides appears to

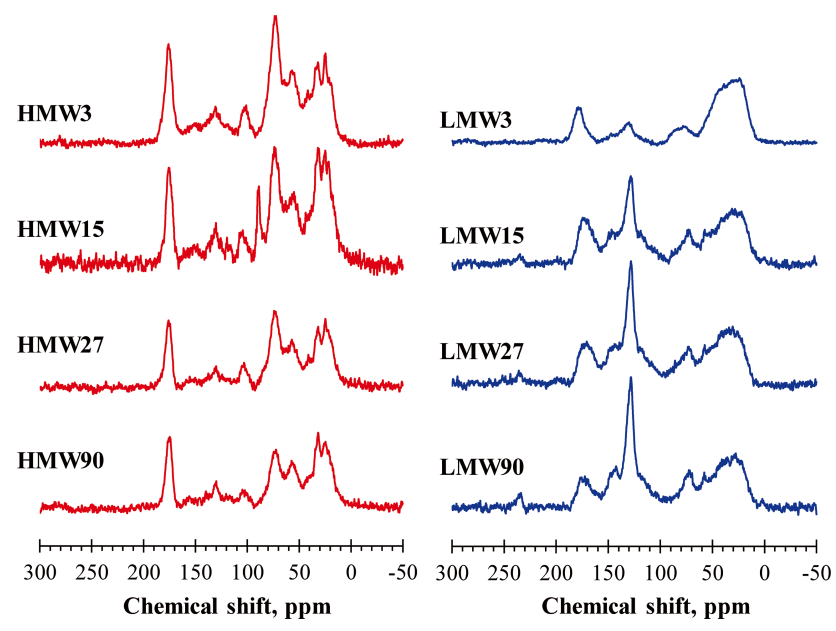

Fig. 5 Solid state CP-MAS ${ }^{13} \mathrm{C}$ NMR spectra of sequentially extracted SWEOMs have decreased, as evidenced by a $1032 \mathrm{~cm}^{-1}$ peak in the FT-IR spectrum for compost after a 90-day period of incubation (Fig. 4(C)). The results of NMR analyses are also consistent with saccharides being lost from HMW (60 - 110 ppm) during the incubation (Table 2; Fig. 5). In addition, low molecular aromatic compounds would be released via the degradation of lignin by anaerobic bacteria. ${ }^{22,23}$ High amounts of lignin compounds contained in bark tips repesent a source of aromatic compounds. ${ }^{17}$ It is likely that biodegradation products were eluted as the LMW fraction during long term incubation. We, therefore conclude that, the structural changes in the HMW and LMW are a result of the activation of anaerobic microorganisms. SWEOM might be produced from compost as metabolites and by-products of anaerobic bacteria, although SWEOM initially contained in the compost is mostly eluted at the initial stage of fertilization.

\section{Gametogenesis assay}

Reproductive growth on gametophyte of brown macroalgae is induced by Fe. ${ }^{5,6}$ Figure 6 shows the percent of mature female gametophytes (S. japonica) incubated in media containing sequentially extracted SWEOMs. Chelate-free and $1 \mu \mathrm{M}$ of EDTA containing media were used as controls. Even though $\mathrm{Fe}$ was added, as shown in Fig. 6, the maturity for a 14-day period was very low in the chelate-free medium ( $\mathrm{ca} .17 \%)$. In the media containing $1 \mu \mathrm{M}$ Fe with EDTA as a chelating agent, the maturity for a 14-day period of incubation reached about $90 \%$.

In the case of media in which Fe was not added, $0-10 \%$ of maturity was observed for both the HMW and LMW. The maturation appeared to be caused by low levels of contaminating iron in the SWEOMs. ${ }^{6}$ In the media in which HMW and $\mathrm{Fe}$ $(1 \mu \mathrm{M})$ were added, $30-50 \%$ of gametophytes were converted to eggs. The difference between the maturity in the case of HMW90 alone (ca. 15\%) and that in which HMW90 and $\mathrm{Fe}$ were added (ca. $30 \%)$ were only $15 \%$. Considering the result of chelate-free condition, the increase could be caused by just Feadded, rather than the effect of HMW90. For the case of added LMW and $\mathrm{Fe}(1 \mu \mathrm{M})$, an average maturity of about $70 \%$ was observed in the LMW3. Although the maturity was decreased to about $30 \%$ of the average value for the LMW15, these values were regained to about $60 \%$ of the average values for the LMW30 and LMW90. The percent maturity in the case of HMW15 with Fe was slightly larger than that of other HMWs, suggesting that an effective chelate agent was distributed in HMW fraction at 15 day of extraction period. Most of the initial contained LMW fraction in compost was eluted at early

Table 2 Composition of carbon species determined by the solid-state CP-MAS ${ }^{13} \mathrm{C}$ NMR spectra for sequentially extracted SWEOMs

\begin{tabular}{|c|c|c|c|c|c|c|c|c|c|}
\hline \multirow{3}{*}{$\begin{array}{c}\text { Range, } \\
\text { ppm }\end{array}$} & \multirow{3}{*}{ Assigned group ${ }^{\mathrm{a}}$} & \multicolumn{8}{|c|}{ Compositions of carbon species, $\%$} \\
\hline & & \multicolumn{4}{|c|}{ HMW } & \multicolumn{4}{|c|}{ LMW } \\
\hline & & 3 & 15 & 27 & 90 & 3 & 15 & 27 & 90 \\
\hline $0-45$ & Alkyl-C & 26.0 & 32.3 & 29.9 & 32.4 & 46.1 & 27.3 & 23.6 & 25.3 \\
\hline $45-60$ & N,O-Alkyl-C & 11.0 & 10.0 & 10.2 & 10.6 & 12.5 & 8.24 & 8.68 & 8.15 \\
\hline $60-110$ & Polysaccharide-C & 32.3 & 30.2 & 30.1 & 25.4 & 12.1 & 16.0 & 17.7 & 17.3 \\
\hline $110-140$ & Aromatic- $(\mathrm{C}-\mathrm{H}, \mathrm{C}-\mathrm{C})$ & 9.45 & 8.55 & 8.64 & 9.65 & 8.82 & 22.1 & 25.3 & 26.8 \\
\hline $140-165$ & Aromatic-(C-O) & 6.07 & 5.20 & 5.14 & 5.58 & 4.49 & 11.3 & 11.7 & 10.8 \\
\hline $165-185$ & Carbonyl-(COO) & 12.9 & 10.4 & 11.5 & 12.5 & 11.4 & 11.3 & 9.69 & 8.11 \\
\hline $185-220$ & Carbonyl-(C=O) & 2.32 & 3.31 & 4.44 & 3.89 & 4.65 & 3.70 & 3.28 & 3.60 \\
\hline
\end{tabular}

a. Alkyl-C, alkyl carbon; N,O-Alkyl-C, alkyl carbon attached to hetero atoms; Polysaccharide-C, alkyl carbon attached to oxygen derived from polysaccharide; Aromatic- $(\mathrm{C}-\mathrm{H}, \mathrm{C}-\mathrm{C})$, aromatic carbon attached to hydrogen or carbon; Aromatic- $(\mathrm{C}-\mathrm{O})$, aromatic carbon attached to oxygen; Carbonyl-(COO), carbonyl carbon in acids, esters and/or amine; Carbonyl-(C=O), carbonyl carbon in quinones. 


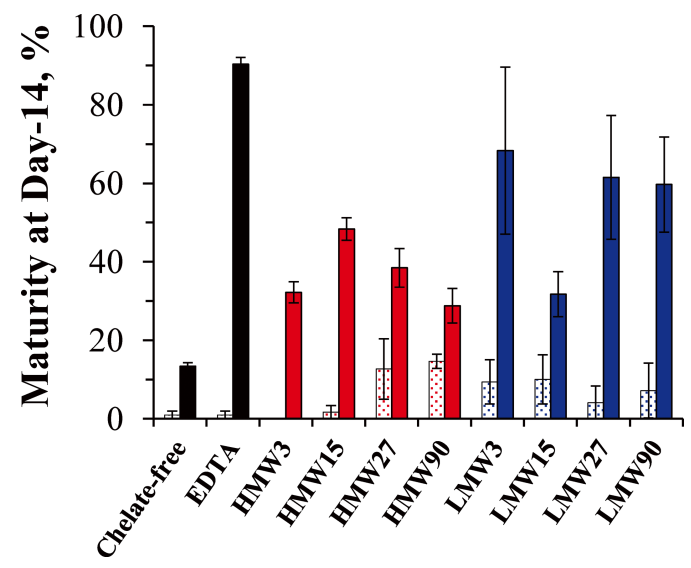

Fig. 6 Effect of SWEOM on oogenesis in female gametophytes of $S$. japonica. The column height denote percent maturities $( \pm$ S.D., $n=3$ ) at a 14-day of incubation period. The concentration of EDTA, HMW and LMW were $1 \mu \mathrm{M}, 5 \mathrm{mg} \mathrm{L}^{-1}$ and $10 \mathrm{mg} \mathrm{L}^{-1}$, respectively. Meshed and filled column indicate Fe-unadded and -added $(1 \mu \mathrm{M})$ conditions, respectively.

extraction stage (Table 1), and LMW15-90 could be supplied by activation of anaerobic bacteria. After 15 days of the extraction period, an effective chelate agent was distributed into the LMW fraction with progressing of the biodegradation. At 15 days of the extraction period, an effective chelate agent was contained in the HMW fraction, because it was not decomposed enough to be distributed to LMW fraction yet. This is a plausible explanation why the maturities changed in the case of HMW1590 and LMW15-90. A 90-day extraction period is considered to a sufficient period to permit the initial restoration of annual brown algae. For brown macroalgae, about one month is required form juvenile sporophytes via gametogenesis and sexual reproduction. ${ }^{1,24}$ The effect of SWEOM on oogenesis was expected, although when SWEOM extracted during a 90day of the incubation period.

\section{Conclusions}

This study confirms that SWEOM can contribute to elute during a 90-day incubation period. The alterations in spectroscopic characteristics for HMW during the incubation period indicate that the concentrations of aromatic compounds and saccharides were decreased, and the aliphatic content of the samples were significantly increased. In contrast, the spectral features of LMW indicate that the aromatic-rich structures in the LMW were altered during incubation. The activity of anaerobic bacteria could be a cause of the structural alterations in the SWEOM, and could contribute to the supply of SWEOM in a long term period of incubation. This study also concluded that SWEOM could continue to be produced from compost as metabolites and by-products of anaerobic bacteria, and, in particular, LMW could be expected to serve as a useful agent to in the restoration of seaweed beds.

\section{Acknowledgements}

We are grateful for the financial support provided by the Nippon Steel \& Sumitomo Metal Corporation (Tokyo, Japan).

\section{References}

1. C. S. Lobban and P. J. Harrison, "Seaweed Ecology and Physiology", 2000, Cambridge University Press, New York.

2. I. Bartsch, C. Wiencke, K. Bishof, C. M. Buchholz, B. H. Buck, A. Eggert, P. Feuerpfeil, D. Hanelt, S. Jacobsen, R. Karez, U. Karsten, M. Molis, M. Y. Roleda, H. Schubert, R. Schumann, K. Valentin, F. Wweinberger, and J. Wiese, Eur. J. Phycol., 2008, 43, 1.

3. M. H. Graham, Ecosystems, 2004, 7, 341.

4. M. H. Graham, Bull. Fish. Res. Agency, 2010, 32, 47.

5. T. Motomura and Y. Sakai, Bull. Jpn. Soc. Sci. Fish., 1981, $47,1535$.

6. H. Iwai, M. Fukushima, T. Motomura, T. Kato, and C. Kosugi, J. Appl. Phycol., 2015, 27, 1583.

7. K. Matsunaga, Y. Suzuki, K. Kuma, and I. Kudo, J. Appl. Phycol., 1994, 6, 397.

8. E. Tipping, "Cation Binding by Humic Substances", 2002, Cambridge University Press, New York.

9. M. Yamamoto, M. Fukushima, E. Kiso, T. Kato, M. Shibuya, K. Otsuka, A. Nishida, and T. Komai, J. Chem. Eng. Jpn., 2010, 43, 627.

10. H. Iwai, M. Fukushima, and M. Yamamoto, Anal. Sci., 2012, 28, 819.

11. H. Iwai, M. Fukushima, M. Yamamoto, T. Komai, and Y. Kawabe, J. Anal. Appl. Pyrolysis, 2013a, 99, 9.

12. H. Iwai, M. Fukushima, and M. Yamamoto, Anal. Sci., 2013, 29, 723.

13. N. Fujisawa, M. Fukushima, M. Yamamoto, H. Iwai, T. Komai, Y. Kawabe, and D. Liu, J. Anal. Appl. Pyrolysis, 2012, 95, 126.

14. M. Fuentes, G. González-Gaitano, and J. M. García-Mina, Org. Geochem., 2006, 37, 1949.

15. M. Fuentes, R. Baigorri, G. González-Gaitano, and J. M. García-Mina, Org. Geochem., 2007, 38, 2012.

16. R. M. Silverstein and F. X. Webster, "Spectrometric Identification of Organic Compounds", 6th ed., 1998, John Wiley \& Sons, Inc., New York.

17. K. H. Tan, "Humic Matter in Soil and the Environment. Principles and Controversies", 2003, Marcel Dekker, Inc., New York.

18. R. L. Warshaw, K. R. Kennedy, and J. E. Henrich, in "Humic Substances: Structures, Properties and Uses", ed. G. Davies and E. A. Ghabbour, 1998, The Royal Society of Chemistry, London, 29.

19. R. S. Houk, H. J. Svec, and V. A. Fassel, "Dynamic Mass Spectrometry”, ed. D. Price and J. F. J. Todd, 1981, Vol. 6, Chap. 19, Heyden, London, 234.

20. J. Buffle and D. G. G. Leppard, Environ. Sci. Technol., 1995, 29, 2169.

21. N. Kloster, M. Brigante, G. Zanini, and M. Avena, Colloids Surf., A, 2013, 427, 76.

22. W. J. Schubert, "Lignin Biochemistry", 1965, Academic Press, New York.

23. P. J. Colberg and L. Y. Young, Appl. Environ. Microbiol., $\mathbf{1 9 8 5}, 49,345$.

24. Y. Suzuki, K. Kuma, and K. Matsunaga, Mar. Biol., 1995, $123,173$. 